\title{
k-Robust Network Design Using Resistance Distance: Case of RocketFuel and Power Grids
}

\author{
Ali Tizghadam, Alireza Bigdeli, Alberto Leon-Garcia \\ School of Electrical and Computer Engineering \\ University of Toronto, Toronto, Canada \\ Email: (ali.tizghadam,alireza.bigdeli,alberto.leongarcia@utoronto.ca
}

\begin{abstract}
This paper reconsiders the problem of robust network design form a different point of view using the concept of resistance distance from network science. It has been shown that some important network performance metrics, such as average utilization in a communication network or total power dissipation in an electrical grid, can be expressed in terms of linear combination of point-to-point resistance distances of a graph. In this paper we choose to have a weighted linear combination of resistance distances, referred to as weighted network criticality (WNC), as the objective and we investigate the vulnerability of different network types. In particular, We formulate a min-max convex optimization problem to design k-robust networks and we provide extension to account for joint optimization of resources and flows. We study the solution of the optimization problem in two different networks. First we consider RocketFuel topologies and Abilene as representatives for service provider networks, and we show gains that can be achieved by optimizing link capacities and flows in RocketFuel topologies and Abilene. In the second experience, we show the application of the proposed optimization problem in designing robust electrical grids.
\end{abstract}

\section{INTRODUCTION}

Robustness and vulnerability analysis are two related problems. In robustness theory, we study the effect of environmental variations in the behavior of a network and we try to design a network in such a way to be insensitive to the environmental changes to the extent possible. In vulnerability analysis we are concerned with bottleneck points of a network. We perturb either different parts of the network or various environmental parameters, and we measure the performance degradation due to the perturbation. In this sense, a more robust network is less vulnerable, since it is less sensitive to the unpredicted changes in network parameters.

It has been already shown that resistance distance [1] can quantify a number of important properties and performance metrics in a network. Physical and electrical interpretations of resistance distance are discussed in [1], [2] and a nice summary of the electrical interpretations of resistance distance is available in [3]. Some of the applications and interpretations of average resistance distance (referred to as network criticality) are discussed in [4], [5]. A common message which is implied by previous research on the concept of resistance distance is the fact that optimizing network criticality (or a variation of it) provides robustness. We can design robust network topologies by minimizing network criticality (average resistance distance). We can also develop robust network control algorithms (such as flow assignment) by controlling the value of network criticality.

In this paper we use a generalized form of network criticality as the main objective and we discuss k-robust network design methods, in which the goal is to keep the network operational at the presence of up to $k$ link failures. We use this approach to evaluate present structure of Internet Service Provider (ISP) networks. Moreover, we investigate the design of robust power grids as another application of the proposed method.

The paper is organized as follows. Section II reviews previous work on resistance distance and network criticality, and introduces necessary notations. In section III we formulate our k-robust optimization problem and show extensions to jointly optimize resources and flows in a communication network. Section IV discusses the application of our k-robust method in studying the vulnerability of RocketFuel ISP networks. We also discuss the design of k-robust power grids using WNC in details. Conclusions are presented in section $\mathrm{V}$.

\section{Resistance Distance And Network CRiticality}

The concept of resistance distance [1] is originally defined in electrical circuits. Consider an electrical circuit with a given topology which is modeled as a weighted undirected graph, where the weight of each link equals the conductance (reciprocal of the resistance) of the link. The point-to-point resistance distance between two nodes $s$ and $d$ (denoted by $\left.\tau_{s d}\right)$ is equal to the equivalent resistance seen between $s$ and $d$. More precisely, if we apply a unit current source between $s$ and $d$, the resistance distance $\tau_{s d}$ equals the voltage drop between $s$ and $d$.

Later it has been shown that there is a close analogy between the behavior of resistive electrical circuits and the theory of random-walks in graphs [2]. While there are different ways to describe this relationship, we choose to use a recently introduced graph metric, the notion of random-walk betweenness centrality [6], to explain how random-walks are related to the electrical circuits. Suppose a random-walker starts wandering around from node $s$ and stops when first reaches at destination node $d$, i.e. node $d$ is an absorbing node. The random-walk betweenness of node $k$ for pair $s-d$, denoted by $b_{s k}(d)$, is defined as the average number of times that the random-walker 
traverses node $k$. The total betweenness of node $k$ is the sum of the contributions of all possible pairs, i.e. $b_{k}=\sum_{s, d} b_{s k}(d)$.

Now, let us define node criticality as the random-walk betweenness of a node normalized by its weight value (randomwalk betweenness divided by the node weight). Likewise, we can define link criticality as the normalized link betweenness. Furthermore, network criticality is defined as the average of point-to-point effective resistances. Let $\eta_{k}, \eta_{i j}$, and $\hat{\tau}$ denote the criticality of node $k$, the criticality of link $l=(i, j)$, and network criticality respectively. It has been shown that the following equations are valid [4]:

$$
\begin{aligned}
\tau_{s d} & =l_{s s}^{+}+l_{d d}^{+}-2 l_{s d}^{+} \text {or } \tau_{s d}=u_{s d}^{t} L^{+} u_{s d} \\
\tau_{s d} & =\frac{b_{s k}(d)+b_{d k}(s)}{W_{k}} \\
\hat{\tau} & =\frac{1}{n(n-1)} \sum_{s, d} \tau_{s d}=\frac{2}{n-1} \operatorname{Tr}\left(L^{+}\right) \\
\eta_{k} & =\frac{b_{k}}{W_{k}}=\frac{n(n-1)}{2} \hat{\tau} \\
\eta_{i j} & =\frac{b_{i j}}{w_{i j}}=n(n-1) \hat{\tau}
\end{aligned}
$$

where $L^{+}=\left[l_{i j}\right]$ is the Moore-Penrose inverse of graph Laplacian matrix L [7], $\mathrm{n}$ is the number of nodes, $\operatorname{Tr}(X)$ denotes trace of matrix $X$, and $u_{i j}=\left[\begin{array}{lllllll}0 & \ldots & 1 & \ldots & -1 & \ldots & 0\end{array}\right]^{t}(1$ and -1 are in $i^{t h}$ and $j^{t h}$ positions respectively).

Equations (1) to (5) show the relationship between randomwalk betweenness and resistance distance. Moreover, according to equations (4) and (5), node criticality $\left(\eta_{k}\right)$ and link criticality $\left(\eta_{i j}\right)$ are independent of the node/link position and only depend on $\hat{\tau}$ which is a global quantity of the network.

Network criticality $(\hat{\tau})$ can capture the effect of topology through the betweenness values. A higher value of the node/link betweenness shows a higher risk (criticality) in using the node/link. Furthermore, one can define node/link capacity as the weight of a node/link, then the higher the weight of a node/link, the lower the risk of using the node/link. Therefore, according to equations (4) and (5) network criticality can quantify the risk of using a node/link in a network which in turn indicates the degree of robustness of the network.

Generally speaking, in a weighted graph network criticality can be interpreted as the total resistance of a corresponding electrical network. Consider a weighted network and build an electrical circuit with the same graph as the original network graph, and with link resistances equal to the reciprocal of link weights. Network criticality is equal to the average resistance distance seen between different pairs of nodes in the electrical circuit representation. A high network criticality is an indication of high resistance in the equivalent electrical circuit; therefore, in two networks with the same number of nodes, the one with lower network criticality is better connected, hence better positioned to accommodate network flows.

It has been shown that $\tau_{s d}$ is a convex function of link weights and $\hat{\tau}$ is a strictly convex function of link weights [4].

\section{A. Interpretations of Network Criticality}

In this section we shed more light on the importance of network criticality in the study of communication networks and power grids by providing some of its interpretations.

1) Average Link Betweenness Sensitivity: We are interested in identifying situations where slight changes in the betweenness of a link or node may cause dramatic changes in the betweenness values elsewhere in a network. Thus a reasonable goal in designing robust network control algorithms is to minimize the changes in the betweenness of different links. It has been shown that $\hat{\tau}$ is proportional to the average of random-walk link betweenness sensitivities (derivative of a link betweenness with respect to its weight) [4]:

$$
\hat{\tau}=\frac{1}{n(n-1)(m-1)} \sum_{(i, j) \in E} \frac{\partial b_{i j}}{\partial w_{i j}}
$$

where $m$ denotes the number of nodes of the graph. Equation (6) states that minimization of network criticality results in minimizing the average sensitivity of link betweennesses with respect to the changes in link weights. Therefore, one needs to control the value of $\hat{\tau}$ to keep the change in the average link betweenness sensitivity below a pre-specified level. Equation (6) also describes one advantage of having low network criticality in the robustness enhancement of the network. Suppose that a node is failing or becoming inaccessible so that it is unable to route the traffic passing through it. If we adapt the routing to minimize the criticality, the result is to adjust the betweenness in such a way that traffic is rerouted to other nodes instead of the impaired one and that the resulting flows provide higher robustness against additional unpredictable deleterious situations.

2) Average Travel Cost: Suppose that there are costs associated with traversing links along a path and consider the effect of network criticality on average cost incurred by a message during its walk from source $s$ to destination $d$. It is shown in [4] that the average incurred cost is the product of network criticality and the total cost of all link weights. Therefore, if we set a fixed maximum budget for the cost of assigning weights to links, then the average travel cost is minimized when network criticality $(\hat{\tau})$ is minimized. In the special case where all links have unit cost, the average travel cost is equal to the average hop length (or average travel time). Hence $\hat{\tau}$ quantifies average path length.

3) Congestion: $\hat{\tau}$ can determine the onset of congestion in a communication network. Let $\lambda$ be the average total input rate of the network, and let the weight of each link be the capacity of the link. It can be shown that the maximum acceptable value of $\lambda$ before the capacity of some link in the network is exceeded (congestion) is upper bounded by the reciprocal of network criticality [4]. One concludes that to delay the onset of congestion to the maximum extent possible, we need to minimize $\hat{\tau}$.

4) Average Network Utilization: Let the link weights be the capacity of the links in the network and a traffic demand matrix is given, then the average network utilization can be expressed as a linear combination of point-to-point resistance distances, i.e. $\bar{V}=\sum_{i j} \beta_{i j} \tau_{i j}$, where $\beta_{i j}$ is a function of traffic matrix entries [5]. Hence, optimizing network utilization is related 
to the optimization of a linear combination of point-to-point resistance distances.

\section{B. Power Dissipation in Electrical Grids}

It can be shown that in a DC-model approximation of a power grid, the average power dissipation of the grid is proportional to $\operatorname{Tr}\left(A L^{+}\right)$, where $A$ is a positive semi-definite matrix [8]. It is easy to verify that the power dissipation can be expressed as a linear combination of point-to-point resistance distances (similar to the case of network utilization).

All of these interpretations show that minimization of a linear combination of point-to-point resistance distances, which will be referred to as weighted network criticality (WNC), is the basis of optimizing many important network performance metrics such as path cost, utilization, and power dissipation. Hence, in the following section we investigate the properties of such an optimization problem.

\section{Weighted NeTwORK CRITICALITY}

We consider a general weighted version of network criticality (WNC) defined as follows.

$$
\tau_{\alpha}=\sum_{i, j} \alpha_{i j} \tau_{i j}, \quad \forall i, j \in N \quad \alpha_{i j}+\alpha_{j i} \geq 0
$$

where $N$ is the set of network nodes. To study the minimization of WNC, we rewrite WNC in matrix form as follows:

$$
\tau_{\alpha}=\sum_{i, j} \alpha_{i j} \tau_{i j}=\sum_{i j} \alpha_{i j} u_{i j}^{t} L^{+} u_{i j}=\operatorname{Tr}\left(U_{\alpha} L^{+}\right)
$$

where $U_{\alpha}=\sum_{i j} \alpha_{i j} U_{i j}$ and $U_{i j}=u_{i j} u_{i j}^{t}$.

It is easy to see that the sum of the rows in $U_{\alpha}$ is zero, and for $\alpha_{i j}+\alpha_{j i} \geq 0 \quad \forall i, j \in N$, it is a symmetric and positive semidefinite matrix. One example of $U_{\alpha}$ for $n=3$ (number of nodes) is given in the following:

$$
U_{\alpha}=\left(\begin{array}{ccc}
\alpha_{12}^{\prime}+\alpha_{13}^{\prime} & -\alpha_{12}^{\prime} & -\alpha_{13}^{\prime} \\
-\alpha_{12}^{\prime} & \alpha_{12}^{\prime}+\alpha_{23}^{\prime} & -\alpha_{23}^{\prime} \\
-\alpha_{13}^{\prime} & -\alpha_{23}^{\prime} & \alpha_{13}^{\prime}+\alpha_{23}^{\prime}
\end{array}\right)
$$

where $\alpha_{i j}^{\prime}=\alpha_{i j}+\alpha_{j i}$.

We now consider minimization of WNC. First we show that the minimization is viable. Using the properties of $\tau_{i j}$ [4], it is easy to verify that the partial derivative of $\tau_{\alpha}$ with respect to link weight $w_{i j}$ is always non-positive and can be obtained from the following equation.

$$
\frac{\partial \tau_{\alpha}}{\partial w_{i j}}=-\left\|F_{\alpha} L^{+} u_{i j}\right\|^{2}
$$

where $F_{\alpha}$ is a matrix such that $U_{\alpha}=F_{\alpha}^{t} F_{\alpha}$. This decomposition is always possible because $U_{\alpha}$ is a positive semidefinite matrix.

Since WNC is a convex function and its derivative with respect to the weights is always non-positive, the minimization of $\tau_{\alpha}$ subject to some convex constraint set is possible.

In formulating the optimization problem, we add a maximum budget constraint to the problem. We assume that there is a cost $z_{i j}$ to deploy each unit of weight on link $(i, j)$. We also assume that there is a maximum budget of $C$ to spend across all network links. This constraint means that $\sum_{(i, j) \in E} w_{i j} z_{i j} \leq C$. Now we can write our optimization problem as follows:

$$
\begin{gathered}
\text { Minimize } \tau_{\alpha} \\
\text { Subject to } \quad \sum_{(i, j) \in E} w_{i j} z_{i j} \leq C \quad, C \text { is fixed } \\
w_{i j} \geq 0 \quad \forall(i, j) \in E
\end{gathered}
$$

Assuming $\Gamma=L+\frac{J}{n}$, and considering the fact that $L=$ $\sum_{i, j} w_{i j} u_{i j} u_{i j}^{t}$ (definition of Laplacian) and $L^{+}=\Gamma^{-1}-\frac{J}{n}$ [9], where $J$ is a square $n \times n$ matrix with all entries equal to 1 , we can write the optimization problem (9) as:

$$
\begin{gathered}
\text { Minimize } \quad \operatorname{Tr}\left(U_{\alpha} L^{+}\right) \\
\text {Subject to } \quad \Gamma=\sum_{(i, j) \in E} w_{i j} u_{i j} u_{i j}^{t}+\frac{J}{n} \\
L^{+}=\Gamma^{-1}-\frac{J}{n} \\
\sum_{(i, j) \in E} w_{i j} z_{i j}=C \quad, C \text { is fixed } \\
w_{i j} \geq 0 \quad \forall(i, j) \in E
\end{gathered}
$$

Note that $U_{\alpha} J=0$, consequently $\operatorname{Tr}\left(U_{\alpha} L^{+}\right)=\operatorname{Tr}\left(U_{\alpha} \Gamma^{-1}\right)$.

\section{A. Network Planning Using Semi-Definite Programming}

Optimization problem (10) (or problem (9)) provides an approach for robust network design via optimal allocation of network link weights to minimize weighted network criticality. Optimization problem (10) can be converted to a semi-definite program (SDP) as stated in the following.

$$
\begin{gathered}
\text { Minimize } \quad \operatorname{Tr}(Y) \\
\text { Subject to } \quad \sum_{(i, j) \in E} w_{i j} z_{i j} \leq C \quad, C \text { is fixed } \\
w_{i j} \geq 0 \quad \forall(i, j) \in E \\
\left(\begin{array}{cc}
L+\frac{J}{n} & U_{\alpha}^{\frac{1}{2}} \\
U_{\alpha}^{\frac{1}{2}} & Y
\end{array}\right) \succeq 0
\end{gathered}
$$

where $\succeq$ means positive semi-definite.

Solving this SDP problem is much faster and can be done with a variety of existing packages (for example see [10]).

\section{B. Robust Network Design: Protecting Against Multiple Link Failures}

The solution of optimization problem (9) or (10) provides a robust network design method via optimal weight assignment; however, it does not necessarily protect the network against multiple link failures. Link failures can be the result of unplanned random failures or due to targeted attacks. In this section we extend optimization problem (9) to account for multiple link failures.

Let $D \in\{0,1\}^{m}$ be a binary matrix representing the location of link failures, i.e. $d_{i j}=0$ for failed link $(i, j)$ and $d_{i j}=1$ for operational ones. We replace the weight matrix $W$ with $D o W$ (o is the Hadamard operator) and redo the optimization of WNC. Now if we would like the network to be robust to up to $k$ link 
failures (we refer to such a network as k-robust network), we need to minimize the following objective function.

$$
\max _{\sum_{i, j} d_{i j}=n-k} \operatorname{Tr}\left(U_{\alpha} L^{+}(D o W)\right)
$$

Note that the above function is convex because it is a pointwise maximum of a set of convex functions. By minimizing this function we find a $k$-robust topology (along with its optimal link weights). Therefore, a general optimization problem to provide a k-robust network can be written as:

$$
\begin{gathered}
\text { Minimize } \quad \max _{\sum_{i, j} d_{i j}=n-k} \operatorname{Tr}\left(U_{\alpha} L^{+}(D o W)\right) \\
\text { Subject to } \quad \sum_{(i, j) \in E} w_{i j} z_{i j} \leq C \quad C \text { is fixed } \\
w_{i j} \geq 0 \quad \forall(i, j) \in E
\end{gathered}
$$

1) Joint Robust Optimal routing and Resource Allocation: While our focus in this paper is not in flow assignment or routing problem, we state that by interpreting weight as capacity, optimization problem (12) can be extended to provide simultaneous solution for k-robust flow assignment (routing) and weight assignment (resource allocation), just by adding the flow conservation equations and link capacity constraints. Let $\gamma_{s d}$ denote the traffic demand between nodes $s$ and $d$, then the conservation of flow for a specific node $k$, can be written as:

$$
\sum_{i \in A(k)} f_{i k}^{(s d)}-\sum_{j \in A(k)} f_{k j}^{(s d)}=\gamma_{s d} \delta(k-s)-\gamma_{s d} \delta(k-d)
$$

where $A(k)$ denotes the set of neighbor nodes of node $k, f_{i k}^{(s d)}$ denotes the flow of link $(i, k)$ for traffic entry between source $s$ and destination $d$, and $\delta(x)$ is Kronecker delta function. Furthermore, the flow of each link should not exceed the weight (capacity) of the link, therefore we can write the optimization for robust joint flow assignment and resource allocation as follows:

$$
\begin{aligned}
& \text { Minimize } \max _{\sum_{i, j} d_{i j}=n-k} \operatorname{Tr}\left(U_{\alpha} L^{+}(D o W)\right) \\
& \text { Subject to } \sum_{(i, j) \in E} w_{i j} z_{i j} \leq C \quad, C \text { is fixed } \\
& w_{i j} \geq 0 \quad \forall(i, j) \in E \\
& \sum_{i \in A(k)} f_{i k}^{(s d)}-\sum_{j \in A(k)} f_{k j}^{(s d)}=\gamma_{s d} \delta(k-s)-\gamma_{s d} \delta(k-d) \\
& f_{i j}=\sum_{s d} f_{i j}^{(s d)} \quad \forall(i, j) \in E \\
& f_{i j} \geq 0 \quad \forall(i, j) \in E \\
& f_{i j} \leq w_{i j} \quad \forall(i, j) \in E
\end{aligned}
$$

There is a variety of methods that can be used to develop algorithms to provide the solution of the above optimization problems (such as dual decomposition and interior point methods); however, in this paper our goal is to present the properties of the solution and its benefits. Therefore, in the following section we focus on solving optimization problem (12) for two important networks.
TABLE I

ROCKETFUEL DATASET ISPS

\begin{tabular}{|c||c|c||c|c||c|c|}
\hline ISP & Routers & Links & $\begin{array}{c}\text { Reduced } \\
\text { Cities }\end{array}$ & $\begin{array}{c}\text { Reduced } \\
\text { Links }\end{array}$ & $\begin{array}{c}\text { Weight } \\
\text { per Link }\end{array}$ & $\begin{array}{c}\text { Total } \\
\text { Weight }\end{array}$ \\
\hline 1755 & 87 & 322 & 18 & 33 & 0.7822 & 51.628 \\
\hline
\end{tabular}

\section{ApPLiCATIONS}

In the first and second parts of this section, we study the application of optimization problem (12) and (13) in Rocketfuel topologies [11] and Abilene network [12] respectively. As another application, in the third part, we discuss the design of robust and sparse power grids.

\section{A. RocketFuel Dataset}

Our first experiment is on real ISP (Internet Service Provider) maps from RocketFuel dataset [11]. We followed the method described in [13] and collapsed the RocketFuel ISP topologies into PoP to PoP connectivity networks. In other words, we consolidated all the nodes within a city into a single node, and aggregated all the links from one city to another one in a single link, where the capacity of the link equals the sum of the capacities of all the original links connecting different sub-nodes between two cities. There are six ISP topologies in RocketFuel dataset, whose topological information are given in [13]. The topologies in RocketFuel dataset do not include the capacities of the links, but we can use OSPF weight information which is provided in RocketFuel dataset to associate compatible capacities using Cisco recommendation as described in [13]. Cisco recommends that the link capacities are proportional to the reciprocal of the weights.

In this paper we work with one of the topologies from RocketFuel dataset (see Table I). We would like to study possible gains we may achieve by replacing present capacity allocation for RocketFuel topologies with the optimal weight (capacity) set obtained as the solution of the proposed k-robust method. In the following experiments we consider 4 different weight sets. First of all, since the networks are real, we already have an initial weight (IW) set in which the weights are proportional to the capacity of the real network. In the second weight set, the total weight (total capacity) of the network is uniformly distributed among all link weights; therefore, we have equal weight allocation $(\mathrm{EW})$. The third weight set which is denoted by OT is the solution of optimization problem (11). This solution is robust in the sense that network criticality is minimized; however, it is not optimized for vulnerability (i.e. failures). Finally, the fourth weight set is the result of optimizing the network for link failure. We consider the case of 1-robust topology weight design using optimization problem (12), and the optimal weight set is denoted by MMTL. In this experiments all the link costs $\left(z_{i j}\right.$ 's) are assumed to be 1 .

Table of Fig. 1 shows the value of network criticality (objective function of optimization problem (11)), and the value of $\max _{\sum_{i, j} d_{i j}=n-k} \operatorname{Tr}\left(U_{\alpha} L^{+}(D o W)\right.$ ) (objective function of optimization problems (12)) for RocketFuel topology 1755. In the figure, these values are denoted by $\hat{\tau}$ and $\hat{\tau}^{i j}$ (the 


\begin{tabular}{|l|c|c|}
\hline & $\hat{\boldsymbol{\tau}}$ & $\max \hat{\boldsymbol{\tau}}^{(i j)}$ \\
\hline IW & 1.9408 & 3.6802 \\
\hline EW & 1.1977 & 1.5478 \\
\hline OT & 1.1013 & 1.3774 \\
\hline MMTL & 1.1239 & 1.3277 \\
\hline
\end{tabular}

Fig. 1. Parameters of Different Optimized Weight Sets for 1755 Network

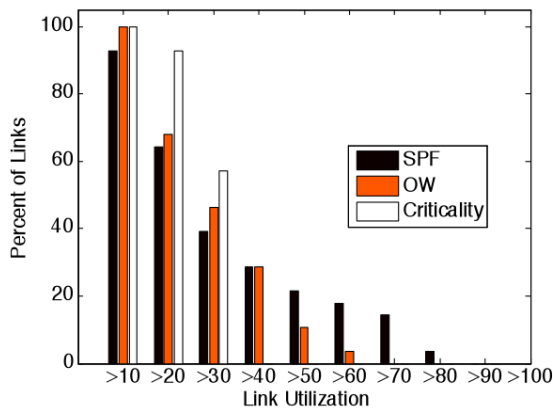

Fig. 2. Abilene Network: Link Utilization for 3 Different Traffic Engineering Methods: SPF, OW, and Network Criticality Minimization

superscript $i j$ shows that $\hat{\tau}^{i j}$ is the value of network criticality when link (i,j) is removed) respectively. It can be easily seen that there is a huge gap between IW and optimized weight assignment for RocketFuel networks. This verifies that our optimizations can significantly improve the vulnerability of the network. For example, according to the table of Fig. 1, the optimal vulnerability parameters of network 1755 show $42 \%$ and $61.4 \%$ improvement respectively comparing with initial weight (IW) case.

\section{B. Optimal Joint Capacity Allocation/Routing for Abilene}

In order to evaluate optimization problem (13), in this section we consider Abilene network [12], since the real traffic matrix traces for Abilene are publicly available (see the website of TOTEM project [14] for a wide range of Abilene traffic traces for different dates). We used one of the available traffic matrices for Abilene, and solved joint optimization problem (13) to find optimal link capacities and link flows simultaneously. We measured the utilization of all the links of Abilene and compared it with the link utilization of two other traffic engineering methods, i.e. SPF (shortest path first) and OW, where OW is a weight optimization tool for Intradomain internet routing protocols. OW algorithm determines the weight of the links in order to utilize the network more efficiently by using tabu search meta-heuristic method [15]. We used TOTEM software package [14] to find the best routes (flow assignment per link) for the given traffic matrix using SPF and OW methods, where the capacities were taken to be the default Abilene's link capacities specified in [12].

Fig. 2 shows the aggregate link utilization for all three traffic engineering approaches. According to Fig. 2, in our method (criticality), there is no link with utilization more than $40 \%$, while in the other two methods (SPF, and OW) we have links

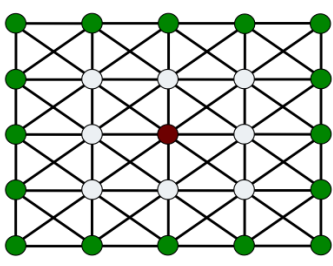

(a)

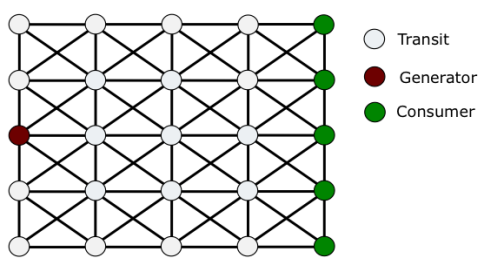

(b)
Fig. 3. Power grids with one Generator Node

with high utilization, which makes the network vulnerable to the future demands (if any). Our simulations revealed that if we shut down a random link in Abilene, in most of the tests both SPF and OW methods will have some links with $100 \%$ utilization, while our method keeps the utilization of all the links always below $70 \%$.

\section{Design of Robust Power Grids}

The concept of (weighted) network criticality has a nice application in power grids. Nowadays the idea of using renewable energy sources has gained considerable attention. Many places with renewable energy (such as places with high wind) are not within the reach of existing power grid network and it is required to extend the existing power grid to the places with renewable energy. Thus, we need to know how to design a robust power network either standalone or as an extension to the existing one. In addition to the robustness, a power grid should be sparse enough, to avoid unnecessary power lines.

As elaborated in section II, the average power dissipation of the grid in a DC-model approximation of a power grid, is proportional to $\operatorname{Tr}\left(A L^{+}\right)$, where $A=<\vec{a} \vec{a}^{t}>$ and $\vec{a}$ is the vector of link electrical currents $(<.>$ denotes time average) [8]. Clearly $A$ is a positive semi-definite matrix; consequently, we can rewrite $\operatorname{Tr}\left(A L^{+}\right)$in the form of WNC (i.e. as a linear combination of $\tau_{i j}$ 's) using equations (1) to (5). Therefore, minimization of power dissipation in power grids results in minimization of WNC. We address the optimization of a power grid network with multiple random independent loads supplied by a generator. For consumer nodes, we specify mean load $\bar{a}_{i}<0$ and the variance $\sigma_{i}^{2}$. At transmission (relay) nodes, the average and variance of the load are zero. At the generator we must have $a_{0}=-\sum_{i \neq 0} a_{i}$. Therefore, matrix $A=\left\langle\vec{a} \vec{a}^{t}\right\rangle$ can be written as:

$$
\left(\begin{array}{cc}
\left(\sum_{i \neq 0} \bar{a}_{i}\right)^{2}+\sum_{i \neq 0} \sigma_{i}^{2} & -\overrightarrow{1}^{t}\left(\vec{a} \vec{a}^{t}+\Sigma\right) \\
-\left(\vec{a} \vec{a}^{t}+\Sigma\right) \overrightarrow{1} & \vec{a} \vec{a}^{t}+\Sigma
\end{array}\right)
$$

We let $\bar{a}_{i}=-1$ and $\sigma_{i}^{2}=\frac{1}{4}$ for consumer nodes in our tests in this section. We consider an $n-b y-n$ grid ( $n$ is an odd number) and we let the generator node be the middle node of the grid and consumer nodes on the border nodes (Fig. 3-(a)), or a middle node in one of the border lines of the grid and consumers on the parallel border (Fig. 3-(b)).

First, we optimize the grid for power dissipation (i.e. we minimize weighted network criticality $\operatorname{Tr}\left(A L^{+}\right)$. The optimization problem is essentially the same as problem (11) with 


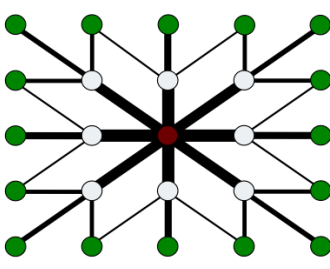

(a)

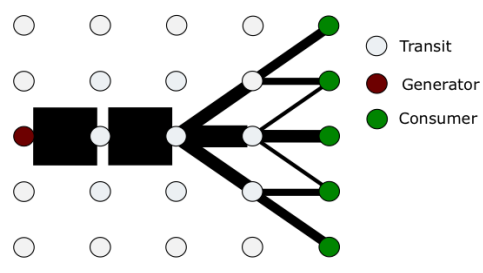

(b)
Fig. 4. Optimal Grid Topologies - Thickness of the lines represent the conductances

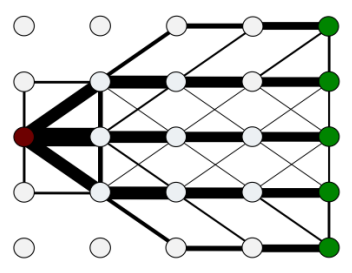

(a)

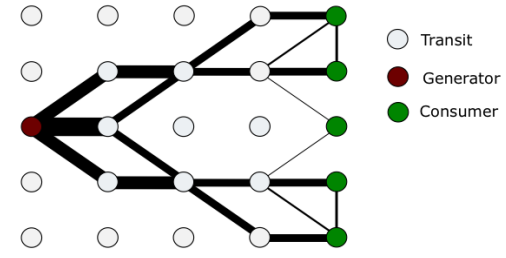

(b)
Fig. 5. (a) Optimal Robust Grid Topology (Link Failure), (b) Optimal Robust Sparse Grid topology

appropriate values of $\alpha_{i j}$, so that $U_{\alpha}=A$. We solved problem (11) for the given values of $U_{\alpha}=A$. The optimal networks are shown in Fig. 4-(a), (b), where the thickness of the lines represent the link weights or line conductances (thicker line has higher conductance). We discuss Fig. 4-(b), since it is more vulnerable and needs attention. Fig. 4-(b) shows that by optimizing weighted network criticality we prune the original grid; however, this network does not provide protection against possible link failures. We can use optimization problem (12) to find a k-robust grid power topology. Fig. 5-(a) shows an example of a 1-robust topology.

We provide one more extension that is particularly useful for the case of power grids in which the network should be sparse enough while preserving robustness. We would like to sparsify the robust topology of 5-(a). Fortunately, there is an elegant study on the context of sparsification using resistance distance. In [16] the problem of finding an sparse version of a network is addressed with the goal of keeping the total resistance distance of the original graph and its sparse version as close as possible. The authors have proposed an algorithm to find such sparse networks. The algorithm works as follows. Suppose $H$ is the sparse version of graph $G$. Choose a random line $(i, j)$ of the network $G$ with probability $p_{i j}$ proportional to $w_{i j} \tau_{i j}$, where $\tau_{i j}$ is the point-to-point network criticality or the resistance distance seen between nodes $i$ and $j$. Add $(i, j)$ to $H$ with weight $\frac{w_{i j}}{q p_{i j}}$, where $q$ is the number of independent samples (we should sum up weights if a line is chosen more than once). We used this algorithm to simplify the optimal robust network of Fig. 5-(a), and the result is shown in Fig. 5-(b).

The network criticality of the sparse topology in Fig. 5-(b) is close to that of the original topology (Fig. 5-(a)) and it is still 1-robust, but the structure that number of active links (power lines) in the topology of Fig. 5-(b) is much less than the original one.

\section{COnClusions}

We investigated the application of network criticality to the design of k-robust networks. We argued that some of the important network performance metrics can be generally expressed as weighted network criticality (WNC), which is in fact a linear function of point-to-point resistance distances. We then constructed an optimization problem to minimize WNC, and discussed a k-robust (min-max) approach in which the network is protected against up to $\mathrm{k}$ link failures. We studied the optimal weight assignment for RocketFuel topologies. We also studied the joint robust assignment of capacities and flows using WNC, and we verified the usefulness of the proposed optimization method by solving the joint resource and flow assignment for Abilene network. Moreover, we showed the application of the min-max optimization problem in the design of robust power grid topologies. We also presented a method to sparsify the robust solution to avoid unnecessary expensive power links.

In our next research, we consider the design of robust networks when the links are directional. Furthermore, we study the case of designing robust networks at the presence of correlated link failures.

\section{REFERENCES}

[1] D. J. Klein and M. Randic. Resistance Distance. Journal of Mathematical Chemistry, 12(1):81-95, December 1993.

[2] P. G. Doyle and J. L. Snell. Random Walks and Electric Networks. Mathematical Association of America, 1984

[3] A. Ghosh, S. Boyd, and A. Saberi. Minimizing Effective Resistance of a Graph. SIAM Review, problems and techniques section, 50(1):37-66, February 2008.

[4] A. Tizghadam and A. Leon-Garcia. Autonomic Traffic Engineering for Network Robustness. IEEE Journal of Selected Areas in Communications (J-SAC), 28(1):39 - 50, January 2010.

[5] A. Tizghadam and A Leon-Garcia. On Traffic-Aware Betweenness and Network Criticality. In INFOCOM Second Workshop on Network Science for Communications (NetSciCom), San Diego, CA, March 2010.

[6] M. Newman. A Measure of Betweenness Centrality Based on Random Walks. arXiv cond-mat/0309045., 2003.

[7] C. R. Rao and S. K. Mitra. Generalized Inverse of Matrices and its Applications. John Weily and Sons Inc., 1971.

[8] JK. Johnson and M. Chertkov. A Majorization-Minimization Approach to Design of Power Transmission Networks. arXiv:1004.2285, September 2010.

[9] Dennis S. Bernstein. Matrix Mathematics. Princeton University Press, 2 edition, 2009.

[10] M. Grant and S. Boyd. CVX: Matlab Software for Disciplined Convex Programming (Web Page and Software). http : //stanford.edu/ boyd/cvx. September 2008.

[11] N. Spring, R. Mahajan, D. Wetherall, and T. Anderson. Measuring ISP Topologies with Rocketfuel. IEEE/ACM Transactions on Networking (TON), 12(1):2-16, February 2004.

[12] Abilene Network. http://www.internet2.edu/network/.

[13] D. Applegate and E. Cohen. Making routing robust to changing traffic demands: algorithms and evaluation. IEEE/ACM Transactions on Networking (TON), 14(6):1193-1206, December 2006.

[14] TOTEM Project (TOolbox for Traffic Engineering Methods). http://totem.run.montefiore.ulg.ac.be/.

[15] B. Fortz and M. Thorup. Increasing Internet Capacity Using Local Search. Computational Optimization and Applications, 29:13-48, 2004.

[16] DA. Spielman and N. Srivastava. Graph Sparsication by Effective Resistances. In Proceedings of the 40th annual ACM Symposium on Theory of Computing (STOC), pages 563-568, 2008. 